Anticipated choice, attention,

\section{and halo effect*}

\author{
JUDSON MILLS, University of Missouri, Columbia, Mo. 65201 \\ and \\ EDGAR O'NEAL, Tulane University, New Orleans, La. 70118
}

An experiment was conducted to clarify the interpretation of a finding by 0 Neal and Mills that the anticipation of making choices about other persons increases the intercorrelation of traits attributed to those persons. An attention interpretation of their finding assumes that greater attention was paid to the choice stimuli and that increased attention increased the intercorrelation of traits. Although the O'Neal-Mills finding was obtained, no support was found for either of the assumptions of the attention interpretation. Ss did not spend longer ranking the choice stimuli, and a successful manipulation of attention did not increase the intercorrelation of the rankings. It was concluded that a desire for certainty about the prospective choice provides the best explanation of the influence of anticipated choice on the halo effect.

The present study was conducted to clarify the interpretation of a recent finding by O'Neal \& Mills (1969) that the anticipation of making choices about other persons increases the intercorrelation of traits attributed to those persons. In the O'Neal-Mills study, male college students ranked female photographs of two different sizes on a number of desirable traits after they had been told that half of the photographs of each size were of promiscuous girls and that in a later experiment they would choose from among the photographs of one size (the choice set) the ones that were of the promiscuous girls. As predicted, the intercorrelation of rankings of the photographs in the choice set was higher than the intercorrelation of rankings of the other photographs.

The hypothesis that the anticipation of making choices about other persons increases the intercorrelation of traits attributed to those persons was based on a theory of choice certainty (Mills, 1968 ). This theory is similar to Jones \& Gerard's (1967) conceptualization of the unequivocal behavioral orientation. It differs from Festinger's dissonance theory $(1957,1964)$ in that it makes predictions about behavior prior to commitment. The basic assumption of choice certainty theory is that people want to be certain when they take an action that it is better than the alternatives. If they are not certain the action is the best one, they will try to increase their certainty; the less their certainty, the stronger will be their desire to increase it. They will also avoid decreasing their certainty. Certainty that an action is best is assumed to be determined by the proportion of cognitions favoring that action; the higher the proportion, the greater the certainty.

* This study was supported by a grant from the National Science Foundation.
People who are faced with a choice between different actions can increase their certainty that the preferred action is the best before committing themselves to it by changing their cognitions about the various alternatives so that more of them favor the preferred action. It follows that individuals who are faced with choices regarding other persons will try to increase their certainty about the choices by changing their cognitions about the other persons so that more of them favor choosing the persons who are preferred. The effect of this should be to increase the intercorrelation of the traits attributed to the other persons or, in other words, to increase the halo effect. ${ }^{1}$ Thus it was predicted that the anticipation of making choices about other persons increases the intercorrelation of the traits attributed to those persons.

An alternative explanation of the results of the O'Neal-Mills study assumes that the higher intercorrelation of the rankings of the choice photographs occurred not as a consequence of a desire for certainty about the prospective choices but simply because the $S$ s paid more aitention to the choice photographs. This attention interpretation may not seem very plausible, since it might be expected that perceptual distortions such as the halo effect would be reduced by an increase in attention to the stimuli. However, it is plausible that the Ss in the O'Neal-Mills study paid greater attention to the choice photographs, and it is possible that, taking more care in their rankings of the choice photographs, they ranked them more reliably - which could have produced the higher intercorrelation for the choice photographs.

The attention explanation of the $O$ 'Neal-Mills finding makes two assumptions: (1) The $\mathrm{Ss}$ paid more attention to the choice photographs, and (2) the increase in attention increased the intercorrelation of the rankings. If the first assumption of attention interpretation is correct, then if the photographs in the choice set are ranked separately from the control photographs, rather than together as in the O'Neal-Mills study, more time should be spent in ranking the photographs in the choice set. This assumes that the more attention that is paid to the photographs, the more time will be spent ranking them. If the second assumption of the attention interpretation is correct, then if the amount of attention is increased by some other means, the intercorrelation of the rankings should increase. These implications of the attention explanation were tested in order to clarify the interpretation of the $O$ 'Neal-Mills finding. METHOD

The procedure of the present study was similar to that used by O'Neal \& Mills (1969). Male college students ranked female photographs of two different sizes on a number of desirable traits after they had been told that half of the photographs were of promiscuous girls and that in a later study they would choose from among the photographs of one size (the choice set) which were the ones of the promiscuous girls. The chief differences were the following: (1) The $\mathrm{Ss}$ ranked the small photographs first and then ranked the large photographs, rather than ranking all the photographs together; (2) some Ss were instructed to do a careful job of ranking the small photographs (careful instructions) and to rank the large photographs as quickly as possible (quick instructions), while others ranked the small photographs under the quick instructions and the large photographs under the careful instructions; and (3) the time spent ranking the photographs was measured.

The Ss were 24 male students in introductory psychology at the University of Missouri and 20 male students in introductory psychology at Tulane University. The Missouri students participated to fulfill a course requirement, while those from Tulane participated in order to receive additional points toward their course grade. An equal number of Ss from each university was assigned randomly to four experimental treatments. In one treatment the small photographs were the choice set and were ranked under the careful instructions, while the large photographs were the control set and were ranked under the quick instructions. In a second treatment the small photographs were the choice set and were ranked under the quick 
instructions, while the large photographs were the control set and were ranked under the careful instructions. In the third treatment the small photographs were the control set and were ranked under the careful instructions, while the large photographs were the choice set and were ranked under the quick instructions. In the fourth treatment the small photographs were the control set and were ranked under the quick instructions, while the large photographs were the choice set and were ranked under the careful instructions.

Upon arriving at the experimental room, the $S$ was seated at a table on which were displayed 16 photographs of female students at another university. Eight of the photographs measured $2^{1 / 4} \times 1 \frac{13}{4}$ in., and the remaining eight were $3^{1 / 4} \times 2^{1 / 2}$ in. The $E$ began by explaining that he was conducting two separate studies. He said the first study had to do with the effect of viewing photographs on the perception of personality traits; the $S$ would be asked to rank the photographs displayed in front of him according to a number of personality traits or attributes.

The second study was described as one which was being conducted for the Counseling Center. The Counseling Center was said to be concerned with coeds who have problems related to sexual promiscuity, and one of their complaints was the feeling that they were marked women, that merely by looking at them other students could tell the extent of their indiscriminate sexual behavior. The $\mathrm{E}$ said the second study had to do with the visible aspects of promiscuity. The $S$ was told that the photographs displayed on the table were of girls who attended a large eastern university and were supplied by the counseling service at that school. The large photographs were said to be of senior girls and the small photographs of sophomore girls. Four of the eight photographs of each size supposedly were of girls who had problems related to promiscuity, while the other four photographs of each size were of girls whose problems were not related to promiscuity. The E mentioned that they were interested in whether the ability to detect promiscuity was greater in the case of younger girls or older girls, so some participants were asked to select which four were promiscuous from among the senior girls and some from among the sophomore girls. The $\mathrm{S}$ was told that in the second study he would be asked either to select from among the large photographs of the senior girls or from among the small photographs of the sophomore girls the four which were of the promiscuous girls.
The E told the $S$ that first he would rank the small photos on a number of different personality traits and then repeat the process with the large photos. He added that he was also interested in the speed with which the photographs were ranked. When the $S$ ranked the small photographs, he was asked either to take all the time he needed to do a really careful job or to work as quickly as he could comfortably do so, and when he ranked the large photographs he was asked either to work as quickly as he could comfortably do so or to take all the time he needed to do a really careful job.

The $S$ ranked the eight small photographs on each of the following traits: artistic, courteous, energetic, intelligent, patient, responsible, sincere, and witty. The $E$ put a card with the name of the particular trait in front of the $S$, asked the $S$ to give him the photograph of the girl who had the most of the trait, asked the same question about the remaining photographs, and so on until all the photographs were ranked. Unobtrusively, the $E$ recorded the amount of time which the $S$ spent ranking the photographs on the various traits. The $S$ then ranked the 16 large photographs on the same eight traits, and the $E$ again recorded the amount of time taken. At the completion of the rankings, the $E$ mentioned to the $\mathrm{S}$ that there was something about the experiment he had not told him and asked him what he thought it might be. None of the Ss' comments indicated suspicion of the instructions. Finally, the E revealed the true nature of the experiment and asked the $S$ not to discuss it with anyone.

\section{RESULTS AND DISCUSSION}

Before examining the data
attention interpretation of the O'Neal-Mills results, it is appropriate to consider whether the same results were obtained in the present study, that is, whether the intercorrelation of the rankings of the photographs in the choice set was larger than the intercorrelation of the rankings of the photographs in the control set. If the O'Neal-Mills finding was not obtained, the question of its interpretation would be irrelevant. As in the previous study, coefficients of concordance, Kendall's W (1948), were computed for the rankings of the photographs in the choice set and also for the rankings of the photographs in the control set. Kendall's W bears a linear relationship to the average Spearman rank-order correlation between all possible pairs of the rankings. The mean $W$ for the choice set was .30. The mean $W$ for the control set was .25. The difference between the choice and control sets was significant beyond the .02 level. ${ }^{2}$ The O'Neal-Mills finding that the anticipation of making choices about other persons increases the intercorrelation of traits attributed to those persons was obtained so the question about its interpretation is relevant.

The attention interpretation of the higher intercorrelation of the rankings for the choice photographs assumes that the Ss paid greater attention to the choice photographs and ranked them more carefully. If this were the case, then one would expect that the Ss would have spent more time ranking the choice photographs than the control photographs. The mean time spent ranking the choice photographs per trait was $44 \mathrm{sec}$. The mean time spent ranking the control photographs per trait was $40 \mathrm{sec}$. Although the time spent ranking the choice photographs was slightly greater, the difference did not approach statistical significance.

A question might be raised about whether the time scores provide a good measure of how carefully the subjects ranked the photographs. An indication that they do is provided by the comparison between the time spent ranking the photographs under the careful instructions and the time spent ranking them under the quick instructions. The mean time spent ranking the photographs per trait under the careful instructions was 54 sec and under the quick instructions, $30 \mathrm{sec}$. The difference was significant beyond the .001 level. If the time scores did not provide a good measure of how carefully the photographs were ranked, then no difference in the time scores would be expected. The difference between the careful and the quick instructions can also be taken as evidence that attention was successfully manipulated. If the careful and quick instructions had not varied attention, no difference would be expected in the time scores.

If the attention interpretation of the O'Neal-Mills finding is correct, then, if attention is increased in some other way, the intercorrelation of the rankings of the persons should be higher. As just mentioned, the evidence from the time scores indicates that the Ss were definitely paying more attention to the rankings under the careful instructions than under the quick instructions. However, the mean $W$ for the photographs ranked under the careful instructions was .28, while the mean $W$ for the photographs ranked under the quick instructions was .27. The difference does not approach significance. 
The results fail to provide any support for either of the two assumptions of the attention explanation of the O'Neal-Mills results. There is no evidence that in the O'Neal-Mills procedure the $\mathrm{Ss}$ paid greater attention to the choice photographs or that increased attention increases the intercorrelation of the rankings. If either of these two effects occurred, they were much too weak to be detectable. It is clear that even if they did occur, their magnitude was far too small in this situation for them to provide an explanation for the higher intercorrelation for the choice photographs. The results indicate that whatever difference there was between attention paid to choice photographs and the control photographs, it was not as great as the difference in attention created by the variation in the instructions under which the photographs were ranked. Yet the difference in the intercorrelation for the photographs ranked under the careful and the quick instructions was smaller than the difference in the intercorrelation for the choice photographs and the control photographs.

By ruling out the attention interpretation as a feasible explanation for the finding that the anticipation of making choices about other persons increases the intercorrelation of traits attributed to those persons, the present study has clarified the interpretation of the O'Neal-Mills finding. The elimination of the attention interpretation leaves the interpretation in terms of a desire for certainty about the prospective choices as the most suitable explanation for the influence of anticipated choice on the halo effect.

\section{REFERENCES}

FESTINGER, L. A theory of cognitive dissonance. Stanford: Stanford University Press, 1957.

FESTINGER, L. Conflict, decision, and dissonance. Stanford: Stanford University Press. 1964.

JONES, E., \& GERARD, H. Foundations of social psychology. New York: Wiley, 1967.

KENDALL, M. Rank correlation methods. London: Griffin, 1948

MILLS, J. Interest in supporting and discrepant information. In R. Abelson, E. Aronson, W. McGuire, T. Newcomb, M. Rosenberg, and P. Tannenbaum (Eds.). Theories of cognitive consistency: A source book. Chicago: Rand McNally, 1968.

O'NEAL, E., \& MILLS, J. The influence of anticipated choice on the halo effect. Journal of Experimental Social Psychology, 1969,5, 347-351.

SIEGEL, S. Nonparametric statistics. New York: McGraw-Hill, 1956 NOTES

1. The halo effect is defined by Jones \& Gerard (1967) as, "The tendency for trait ratings to show a higher intercorrelation than would be revealed by more objective measurement [p. 713]."

2. Statistical significance was tested throughout by means of the WiIcoxon matched-pairs signed-ranks test (Siegel, 1956). All $\mathrm{p}$ values are two-tailed. to perform prior to his terminating his participation in the experiment. This measure, designated "trials to termination (TTT), " was considered to be analogous to measures of $R E$ e mployed in instrumental conditioning.

Reasoning from the widely accepted view that $K R$ in perceptual-motor tasks affects performance in much the same manner as reinforcement in conditioning (e.g., Bilodeau \& Bilodeau, 1961), Black \& Black (1970) sought to determine if schedule of $\mathrm{KR}$ of TOT on the pursuit-rotor affected TTT in a manner similiar to the effect of reinforcement schedule on $\mathrm{RE}$ in instrumental conditioning. Two groups of $S$ s were given an initial series of 20 trials, during which one group (Group CKR) was correctly informed of their TOT following each trial, while the other group (Group PKR) was given TOT following half of the trials. Subsequently, KR of TOT was discontinued for both groups, and TTT was found to be significantly greater for Group PKR than for Group CKR. This result was considered analogous to the partial reinforcement effect in instrumental conditioning and was interpreted as further extending the empirical parallel between the effects of $\mathrm{KR}$ and those of reinforcement.

The present study was concerned with the question of whether or not shifts in the "quality" or "reward value" of informative feedback (IF) affects pursuit-rotor performance in the same way that shifts in magnitude of reward affect performance in instrumental conditioning. In a recent review of instrumental and differential conditioning studies in which reward magnitude was shifted, Black (1968) concluded that both incremental and decremental shifts in reward result in rapid corresponding shifts in performance. In addition, downward shifts in reward magnitude appeared to result in a reduction in performance to a level lower than that of control Ss which had always received the smaller reward. On the other hand, incremental shifts appeared to result in an improvement in performance but not to a level beyond that of Ss which had always received the larger reward. Thus, "negative contrast effects (NCE)" but not "positive contrast effects (PCE)", were typically obtained.

In the present study Ss who were trained on the pursuit-rotor received IF, which indicated that their performance was either "good" or "poor" relative to a fictitious criterion. Following an initial series of trials IF was abruptly shifted upward or downward in an attempt to determine if subsequent TTT showed 